\title{
Reasons and Solutions for Chinese Undergraduates' Typical English Pronunciation Errors*
}

\author{
Shiqian Zhou \\ The School of Foreign Languages \\ Wuhan University of Technology \\ Wuhan, China
}

Chuang Chen

The School of Foreign Languages Wuhan University of Technology

Wuhan, China

$$
\text { Lu Zhao }
$$

The School of Foreign Languages Wuhan University of Technology Wuhan, China

\author{
Yifan Wang** \\ The School of Foreign Languages \\ Wuhan University of Technology \\ Wuhan, China \\ **Corresponding Author
}

Yue Ban

The School of Foreign Languages

Wuhan University of Technology

Wuhan, China

Xiaoqing Zhang

The School of Foreign Languages

Wuhan University of Technology

Wuhan, China

\begin{abstract}
This paper analyzes the reasons for Chinese undergraduates' typical English pronunciation errors comprehensively. Considering the research results of this team in 2015, the current language learning environment and the features of contemporary English pronunciation teaching in colleges, and through related literature review, this paper puts forward the solutions. Teachers should adopt effective solutions according to the reasons for students' errors and lead them to improve the pronunciation and avoid the negative transfer impact.
\end{abstract}

Keywords-dialects; language transfer; English
pronunciation

\section{INTRODUCTION}

Odlin proposes that language transfer refers to the effect of the characteristics and generality between objects and other completely or incompletely acquired languages [1]. Language transfer theory has played an important role in the study of second language acquisition (SLA). The development of behaviorist empirical study and mentalist epistemology in the 20th century have explained reasons for and effects of first language (L1) transfer for linguists from the aspects of language, psychology, cognition, society etc. [2]. Sound pattern theory explores the reasons from the perspectives of phonological segmentation, positional features, allophones etc. of languages and dialects [3]; Pi Liansheng points out the influence of surroundings including schools, families etc. on study [4]. All the previous study is

*This paper is supported by Wuhan University of Technology Independent Innovation Research Fund and supervised by Professor Zhu Hanxiong. Project No. 2016-WY-A1-01. of great importance in studying the transfer impact of Chinese dialects on college students' English phonetic learning.

In China, the research on the transfer impact of Chinese dialects on English pronunciation has been developing. The comparative study of English and Chinese phonetics has progressed since the 1940s when scholars including Zhao Yuanren, Lv Shuxiang etc. devoted some attention into it. A vast number of works on it have appeared in the 21 st century and it was booming in around 2010 [5]. A Comparison of the Main Features of the Phonetic Systems of Chinese and English by Professor Gui Cankun compares Chinese and English pronunciation and intonations [6]; English Suprasegmental Phoneme and Its Distinguishing Function [7] and A Comparison Between English and Chinese Syllable Structures [8] by He Shanfen study English pronunciation and contrast English and Chinese syllable structures, which provides a new perspective for exploring reasons for Chinses college students' typical English pronunciation errors. However, both the comparative study of English and Mandarin pronunciation popular in the 20th century and the contrastive study of English and Chinese dialect pronunciation in the 21st century [5] mostly focus on the language transfer impact rather than its deeper reasons, not to mention solutions to the negative transfer impact.

Reform of college curriculum focuses equally on listening, speaking, reading and writing in place of the traditional pattern emphasizing reading and writing. Although pronunciation teaching is still slightly overlooked, the better overall teaching quality has improved the English pronunciation of contemporary college students. The 
development of the Internet has facilitated language communication among different regions and provided more abundant English learning resources, which has also improved the English pronunciation. The popularization of Mandarin has lessened the negative transfer impact of Chinese dialects and therefore reduced the difficulty of learning English pronunciation. All these factors have diversified the transfer impact of Chinese dialects on English pronunciation and types of pronunciation errors and their reasons. This study will enhance the understanding of the reasons for college students' typical English pronunciation mistakes and corresponding effective solutions.

\section{RESEARCH DESIGN}

\section{A. Research Objects}

This research covers all the 103 freshmen majoring in English in The School of Foreign Languages, Wuhan University of Technology.

\section{B. Research Methods}

The research was conducted in two phases, through recording and questionnaires. The questionnaires were filled in in Phase 2 during their final test of English Pronunciation Course. Combining with the questionnaire results, this paper proposes the factors influencing students' English pronunciation and solutions. All the tables mentioned in this paper are presented in another paper by this team.

\section{Research Materials}

There are 14 questions in the questionnaire distributed in Phase 2, including students' background information and views of the Course, which helps to think about the pros and cons of the Course from the perspective of students.

\section{ANALYSIS OF THE QUESTIONNAIRE RESULTS}

In this research, 103 questionnaires, $100 \%$ of which are valid, have been distributed and collected. The questionnaire consists of 14 multiple-choice questions whose answers are input into IBM SPSS Statistics (Version 22), a professional software for statistics and data analysis, to produce statistical tables with various statistical methods. Through analyzing the statistics, this paper concludes the elements affecting students' pronunciation and proposes corresponding solutions.

\section{A. Statistics and Analyses of the Questionnaire Results}

(1) How many dialects can you speak besides Mandarin?
A. Only Mandarin.
B. One dialect.
C. Two dialects.
D. More than two dialects.

Analysis: $70.9 \%$ of all students choose B, which exhibits that most students can speak only one dialect.

(2) What do your parents usually speak when talking to you, the dialect or Mandarin?
A. Dialect.
B. Mandarin.

C. The proportions of speaking them are roughly equal.

Analysis: $68.0 \%$ of all students choose A, which means that most parents speak the dialect when talking to their children. Only $9.7 \%$, the lowest percentage, of students choose $\mathrm{C}$, while most students choose A or B, which exhibits the undiversified type of language parents adopt when talking to their children.

(3) What did you mostly speak in your growth before entering the University, the dialect or Mandarin?

A .Dialect.

B. Mandarin.

C. The proportions of speaking them are roughly equal.

Analysis: $46.1 \%$ of all students choose B while $40.2 \%$ choose A, which shows that in their growth the largest portion of students speak mostly Mandarin and the second largest portion speak mostly the dialect. In Class 1 and 3, $48.0 \%$ and $50.0 \%$ of students choose A respectively, both higher than the percentages of students choosing others within each class, which shows that the two classes have the largest portions of students speaking dialects in their growth within each one; in Class 2 and 4, 61.5\% and 64.0\% of students choose B respectively, which shows that most students in the two classes speak mostly Mandarin during their growth.

(4) Do you think whether your dialect (including Mandarin) has influence on your English pronunciation?
A. Yes, it has very strong influence.
B. Yes, it has much influence.
C. Yes, it has some influence.
D. No.

Analysis: $37.9 \%$ and $35.9 \%$ of all students choose $\mathrm{C}$ and $\mathrm{D}$ respectively, both higher than the percentage of students choosing A or B, which indicates that most students think dialects have some or even no influence on the English pronunciation. This does not correspond with the research results, which therefore manifests most students' lack of realization of Chinese dialects' impact on English pronunciation.

(5) Do you like the English Pronunciation Course?
A. Yes, very much.
B. Yes.
C. Just so-so.
D. No.

Analysis: $38.8 \%$ and $47.6 \%$ of all students choose A and $\mathrm{B}$ respectively, which shows that most students like the pronunciation course. In Class $1,52.0 \%$ of students choose A while nobody chooses $\mathrm{C}$ or $\mathrm{D}$, which exhibits that the Course is more popular with Class 1 than with any other class. In Class 3, only $30.8 \%$ of students choose A, lower than that in any other class, while only $19.2 \%$ choose $\mathrm{C}$, higher than that 
in other classes, which implies that students in Class 3 least like the Course.

(6) Do you like your pronunciation teacher?
A. Yes, very much.
B. Yes.
C. Just so-so.
D. No.

Analysis: $76.7 \%$ of all students choose A while only $2.9 \%$ choose $\mathrm{C}$ and none chooses $\mathrm{D}$, which indicates the enormous popularity of pronunciation teachers. In Class 2 only $53.8 \%$ of students choose A, lower than that in any other class and even $\mathrm{C}$ is chosen by some, which implies that the pronunciation teacher is the least welcome in Class 2.

(7) How do you think of the pronunciation of your pronunciation teacher?
A. Very standard.
B. Nearly standard.
C. Just so-so.
D. Not standard.

Analysis: $82.5 \%$ of all students choose A whose percentage in every class is also high, which shows that most students think the pronunciation of pronunciation teachers standard.

(8) How is your attention in the Course?
A. Very concentrated.
B. Basically concentrated.
C. Just so-so, sometimes distracted.
D. Not concentrated.

Analysis: $63.1 \%$ of all students choose B, which indicates that students' attention in class is mostly good. In Class 1, 32.0\% of students choose $\mathrm{A}$, higher than that in other classes, and the sum of the percentages of students choosing $\mathrm{A}$ and $\mathrm{B}$ is higher than that in any other class, which suggests that students in Class 1 have the best attention in class. In Class 3, 26.9\% of students choose C, higher than that in other classes and accounting for $41.2 \%$ of all the students choosing $\mathrm{C}$, which implies that students in Class 3 have the scantest attention.

(9) Do you think whether the Course is effective in improving your English pronunciation?
A. Yes, it is very effective.
B. Yes, it is effective.
C. Yes, it is slightly effective.
D. No, it is not effective.

Analysis: $48.5 \%$ of all choose A, higher than that of other choices, but still less than $50 \%$, which indicates that most students do not feel the Course effective enough. In Class 3,
$65.4 \%$ of students choose B, accounting for $42.5 \%$ of all choosing it, both higher than those in other classes. And $26.9 \%$ of students choose A, accounting for $14.0 \%$ of all students choosing this one, both lower than those in other classes, which shows that students in Class 3 are least confident in the effectiveness of the Course.

(10) Do you think whether English pronunciation is important?
A. Yes, it is very important.
B. Yes, it is Important.
C. Just so-so.
D. No, it is not important.

Analysis: $95.1 \%$ of all students choose $\mathrm{A}$ and the percentage of students choosing it in every class is nearly the same, which indicates that nearly all students have realized the importance of English pronunciation.

(11) How do you think of your English pronunciation?
A. Very standard.
B. Nearly standard.
C. Just so-so,
D. Not standard.

Analysis: $42.7 \%$ of all choose C, higher than that of other choices, but still less than half. $39.8 \%$ choose B, only less than that of students choosing $\mathrm{C}$, which implies students' low confidence in their pronunciation. In Class $4,50 \%$ of students choose B, accounting for $31.7 \%$ of all choosing it, both higher than those in other classes, which shows that students in Class 4 are the most confident about their pronunciation among the four classes.

(12) When speaking English, can you realize your pronunciation errors?
A. Yes, I can realize all errors.
B. Yes, I can realize most errors.
C. Yes, I can realize some errors
D. No, I can realize few errors.

Analysis: $52.4 \%$ of students choose $\mathrm{C}$ and $39.8 \%$ choose $\mathrm{B}$, which indicates that most students think they are not sensitive enough to their own pronunciation errors. In Class $2,65.4 \%$ of students choose $\mathrm{C}$, accounting for $31.5 \%$ of all choosing it, both higher than those in other classes. Furthermore, the sum of the percentages of students choosing A and B is lower than that in other classes, which suggests that students in Class 2 think they are least sensitive to their own pronunciation errors.

(13) When hearing others speaking English, can you realize their pronunciation errors?
A. Yes, I can realize all errors.
B. Yes, I can realize most errors. 
C. Yes, I can realize some errors

D. No, I can realize few errors.

Analysis: $53.4 \%$ of all choose B, which shows that students are rather confident in finding out others' pronunciation errors.

(14) After class, how long do you spend practicing your pronunciation every week?

A. Over 5 hours.

B. 2 to 5 hours.

C. Shorter than 2 hours.

D. Never.

Analysis: $49.0 \%$ of all choose C and $38.2 \%$ choose B, which shows that the time students have spent practicing pronunciation is not enough. In Class $4,57.7 \%$ of students choose B, accounting for $38.5 \%$ of all choosing this one, both higher than those in other classes, which indicates that students in this class have the most practice. However, $19.2 \%$ of students choose D in Class 4, also higher than that in other classes, which implies the wide gap between the lengths of time students have spent practicing pronunciation in Class 4.

\section{B. Reasons}

1) Dialects: Results of Questions 1 to 4 show that many college students have underestimated the transfer impact of Chinese dialects on English pronunciation, which makes it difficult to find the suitable solutions. The recording analyses show that Chinese dialects have obvious transfer impact on students' English pronunciation. Furthermore, the underestimation in fact might be more severe than what this research shows. For example, a student from Shandong claimed he cannot speak Shandong dialect, but his recording analysis shows features of Shandong dialect in his English pronunciation. Learners can achieve positive transfer impact when the disparity between L1 and the second language is small [9]. Since Chinese and English have markedly different articulating manners and habits, Chinese dialects will exert negative transfer impact on students' English pronunciation.

2) Attitudes: Results of Questions 5 to 9 show that most students like the Course and teachers, but classes are different in the attention and the confidence in the effectiveness of this Course in improving their English pronunciation. On the whole, Class 3 least like the course and have the scantest attention. Attitudes are quite significant for learning pronunciation and play an important role in initiative and willpower to overcome hardship [10]. The tendency or avoidance, like or dislike, acceptance or refusal are all the outward manifestation of one's attitude [4]. If students like the Course and teachers, they will have a positive attitude toward learning pronunciation. Otherwise, they will have a negative attitude and hence the ineffectiveness.
3) Consciousness: Results of Questions 10 to 14 show that most students have realized the importance of English pronunciation; students in classes except Class 4 do not have much confidence in their English pronunciation; most students think they are not sensitive to their own pronunciation mistakes, but they think they are to others'; on the whole, students have spent inadequate time practicing pronunciation after class, and there are polarizing differences in it among classes and among students within each class. The power of consciousness determines success or failure [11]. Therefore it matters greatly to learning a foreign language.

Noteworthily, Questions 11 to 13 only explore reasons for pronunciation errors from the perspective of students rather than the fact. Although $39.8 \%$ of students chose "nearly standard" in Question 11, it does not mean that their pronunciation is really nearly standard. Instead, it only shows that they have better self-feeling. Consciousness has limitations, and it might be false or uncertain [12]. Students' self-feeling of their pronunciation is not necessarily correct, and therefore teachers' guidance is indispensable. In general, students think they are more sensitive to others' pronunciation mistakes, which does not necessarily indicate double standards of recognition, however. Their actual recognition ability should be evaluated by listening tests.

4) Environment: Results of Questions 2 and 3 show that most students speak dialects in the communication with their parents as well as in their growth most of the time. Consequently, Chinese dialects will exert transfer impact on their English pronunciation. The environmental factors influencing study include study place, family environment, Internet media etc. [4]. But Chinese students barely have a good English learning environment. The results of Question 14 show that most students scarcely practice English pronunciation after class, hence they lack English pronunciation learning atmosphere. Students' language sense and pronunciation proficiency both come from the learning environment. "Language environment is necessary for language learning [13]." The lack of English environment will cause ineffectiveness in learning pronunciation.

5) Culture: Language and culture are integrated and inseparable [13]. English belongs to Indo-European language family while Chinese belongs to Sino-Tibetan language family. They have lots of differences in both language and culture. Education in China has traditional Chinese cultural features, and the two languages have distinctly different phonetic systems. For example, some English phonemes do not exist in Chinese. Thus Chinese students face many difficulties in learning English pronunciation. There are also differences between the two cultures in conventions and faith, so when faced with a language whose culture is far different from L1, Chinese students' interest cannot be easily aroused, hence the ineffectiveness in learning English pronunciation.

6) Individual: Participants come from different 
provinces and families, and have different levels of English education and pronunciation. Some students graduated from foreign languages schools and some have attended English training courses, while the MET in some provinces excludes listening and thus the time students there have spent practicing English in the senior high school is far less than that in other provinces. Therefore students' English pronunciation is also influenced by individual factors. Different intelligence leads to students' different information input [10]. Due to the education levels and backgrounds, students of minorities often show weaker performance on learning a foreign language [14]. The same pronunciation course exerts different influence on different students. Students with a high learning ability master English pronunciation better than those with a low one, and students with more practice after class learn it better than those without practice.

\section{Solutions}

1) Emphasize the transfer impact of Chinese dialects on English pronunciation: The research shows obvious transfer impact of Chinese dialects on English pronunciation. As Table 6 manifests, the FME rate of /1/ does not change much, and many students still mix /n/ and /l/ after the Course, which means that students tend to transfer the manners of articulating Chinese dialects to English pronunciation. This requires teachers to emphasize the transfer impact of Chinese dialects on English pronunciation, to be aware of students' pronunciation errors caused by the negative transfer impact of Chinese dialects, and to correct them in class.

2) Correct pronunciation errors according to the features of each dialect: The statistical analyses show that for students from different dialect regions, the phonemes easily mispronounced or hard to correct are different, while for those from the same dialect region, their English pronunciation errors tend to be similar. For example, in Table 9, the error rates of $/ \mathrm{t} / \mathrm{in}$ Northern Sub-dialect Region increase rather than decrease after the Course, which means that teachers have not found the common mistakes of students from this sub-dialect region or have not corrected them specifically. Chinese has a vast number of dialects every one of which has its distinctive features, which requires teachers to correct errors according to the features of each dialect and to highlight those error-prone phonemes.

3) Adopt the teaching method of contrasting English and Chinese pronunciation: This research indicates that many English pronunciation errors are caused by negative impact of Chinese dialects. For example, in Tables 7 and 8, there are students in every sub-dialect region mixing $/ \mathrm{n} /$ and /y/ because many Chinese dialects do not distinguish front nasal final from back nasal final. Therefore the correct articulating manners should be emphasized so as to alleviate the negative transfer impact of Chinese dialects. Language comparison is conducive to overcoming the interference of
L1 in learning pronunciation of a foreign language [15]. Teachers should compare error-prone phonemes with the correct ones and emphasize the difference to help students build a comparative memory. Tan Qunying proposes that students should be classified into several groups according to the sub-dialect regions and pronunciation errors, and the comparison should be enhanced through recordings, pictures, videos, dubbing etc. [16]. This method can boost effectiveness of the Course to a large extent.

4) Create personal pronunciation records: The research shows that students have so many different pronunciation errors that teachers can hardly correct them all. For example, in Table 16, the mispronunciation of /vo/ has not improved much after the course, which indicates the ineffective teaching during which teachers might have only corrected the FME but ignored the other errors. Pronunciation records should be created for freshmen according to phonetic tests [2]. By analyzing each student's pronunciation errors according to the test, teachers can know his error-prone phonemes and thus help him correct specifically. Phonetic tests should be taken again after the course. By comparing the two test results, remaining pronunciation errors can be found, and suggestions together with comparative reports will be given to students. Apart from the phonetic tests for freshmen, students in other grades should also be tested once a year for the comparative feedback so as to help them better know their pronunciation and thus practice it by themselves.

5) Increase the interestingness of the Course: Results of Question 3 show that students in Class 3 have the scantest attention in class, and some even get distracted, which means that a portion of students in this class are less interested in the Course or have a laxer learning attitude. One's attitude may change through outside stimulation [10]. Teachers should try to lighten the class atmosphere and thus increase students' interest. By playing humorous English movies and assigning group work to encourage participation, for example, a good English learning atmosphere can be established and students' upbeat attitude toward learning pronunciation can thus be fostered.

6) Reinforce pronunciation imitation and practice through the rich multi-media resources: Results of Question 14 shows that most students barely practice pronunciation after class. Some error-prone phonemes may be forgotten due to lack of practice, which indicates students' improficiency in their right articulating manners. "Language imitation is one of the most effective way of learning a language." [17] Teachers should use multimedia equipment to play original English news or movies to enhance students' sensibility to original English. Also, teachers should assign homework such as movie dubbing and acting English movies, which offers students access to original English and helps them become more familiar with the articulating manners of English, hence a better language sense. 


\section{CONCLUSION}

Pronunciation is fundamental to English learning, and only with it well mastered can students improve their listening, speaking, reading and writing skills. In daily communication, pronunciation plays an important role and deeply influences students' confidence. Therefore, pronunciation teaching should be emphasized. Pronunciation training is a long and difficult process. Teachers and students should, after realizing the transfer impact of Chinese dialects on English pronunciation and combining pedagogy, psychology and phonetics, realize the deep reasons for pronunciation errors, highlight the habits of articulating dialects, attitudes to study, adjustment of consciousness, influence of environment and individuality, and adopt corresponding solutions. In the classroom teaching, teachers should correct pronunciation errors of students from different dialect regions with different approaches. They should adopt the teaching method of contrasting English and Chinese pronunciation, create personal pronunciation records, and increase the interestingness of the course. As for students, they should create a positive atmosphere of English learning, practice the correct pronunciation conscientiously through the rich multi-media resources, and cultivate an upbeat attitude to and good habits of learning English pronunciation, so as to better deal with their pronunciation errors exerted by the negative transfer impact of Chinese dialects.

\section{REFERENCES}

[1] T. Odlin, Language Transfer: Cross-Lingusitic Influence in Language Learning. Cambridge: Cambridge University Press, 1999.

[2] W. Shu, An Investigation of Negative Transfer Impact of Chinese Dialects on English Pronunciation and the Solution -- Taking the English Majors of Zhongshan Institute in University of Electronic Science and Technology as an Example, Journal of Guangxi College of Education, 2013, pp. 80-82.

[3] F. Shi, Vowel Patterns of Beijing Dialect, Nankai Linguistics, 2002, vol. 1, pp. 30-32.

[4] L. Pi, Educational Psychology, 4th ed., Shanghai: Shanghai Educational Publishing House, 2004. pp. 107-168.

[5] C. Chen, Y. Ban, L. Zhao, and X. Zhang, An Investigation of Transfer Impact of Chinese Dialects on English Pronunciation, 2nd International Conference on Social Science, 2015, pp.524

[6] C. Gui. A Comparison of the Main Features of the Phonetic Systems of Chinese and English, Modern Foreign Languages, 1978, pp.44-50.

[7] S. He, English Suprasegmental Phoneme and Its Distinguishing Function, Journal of Foreign Languages, 1989, pp.44.

[8] S. He, A comparison between English and Chinese syllable structures, Foreign Languages Research. 1997, pp.64.

[9] R. Ellis. The study of second language acquisition. Oxford: Oxford University Press, 1994, pp.327-328.

[10] D. Tao. Theory and research on learning attitude. Guangzhou : Guangdong People's Publishing House, 2001, pp. 128-141.

[11] S. Pan. Consciousness-research on psychology. Beijing : The Commercial Press, 1998, pp. 55.

[12] Y. Huo. Consciousness psychology. Shanghai: Shanghai Educational Publishing House, 2006, pp. 190.

[13] H. Wang. Study on the spoken English fossilization for English majors, Journal of Henan Mechanical and Electrical Engineering College, 2016, pp. 71.
[14] S. Glock, C. Kovacs. Educational psychology: using insights from implicit attitude measures, Educational Psychology Review, 2013, pp.503.

[15] W. Fang. Contrastive linguistics on Chinese and English [J]. Foreign Languages Research, 1989, pp. 1.

[16] Q. Tan. The application of the method of contrasting dialects and Mandarin in Mandarin teaching in universities for nationalities---a case study of Guangxi dialects, Time Education, 2014, pp. 36.

[17] Y. Teng. Remove the mother tongue flavor of your English pronunciation - on how to overcome the negative transfer impact of Chinese dialects on English, Overseas English, 2010, pp. 162. 